\title{
Identifying large extra dimensions in dilepton and diphoton final states at the Large Hadron Collider
}

\author{
I.A. Serenkova ${ }^{* a}$,A.V. Tsytrinov ${ }^{b}$ and A.A. Pankov ${ }^{c}$ \\ Abdus Salam ICTP Affiliated Centre at the Gomel State Technical University, 246746, Belarus \\ ${ }^{a}$ E-mail: inna.serenkovalcern.ch \\ ${ }^{b}$ E-mail: tsytrinegstu.by \\ ${ }^{c}$ E-mail: pankoveictp.it
}

\begin{abstract}
Arkani-Hamed, Dimopoulos and Dvali (ADD) proposed a model in which gravity propagates freely in $d$ extra, compact spatial dimensions. The prospects of discovery and identification of large extra spatial dimensions effects in the process of lepton and photon pair production at the Large Hadron Collider (LHC) were studied. These effects can be found by the specific behavior of the invariant mass distributions of the lepton and photon pairs. Identification of the effects under study in lepton and photon pairs production can be performed with center-egde asymmetry. Discovery and identification reach on the mass scale parameter $M_{S}$ can be obtained for graviton Kaluza - Klein towers in lepton and photon pair production processes at the LHC.
\end{abstract}

XXII International Baldin Seminar on High Energy Physics Problems, September 15-20, 2014

JINR, Dubna, Russia

${ }^{*}$ Speaker. 


\section{Introduction}

Theories of low-scale quantum gravity featuring large extra spatial dimensions (LED) have attracted considerable interest because of their possible observable consequences at existing and future colliders. In scenario, proposed by Arkani-Hamed, Dimopoulos, and Dvali [1], the fermions and gauge bosons of the Standard Model (SM) are confined to the three ordinary spatial dimensions, which form the boundary ("the brane") of a space with $d$ compact spatial dimensions ("the bulk") in which gravitons alone can propagate. In this model, the Planck scale is lowered to the electroweak scale of $\mathscr{O}(1 \mathrm{TeV})$, which is postulated to be the only fundamental scale in nature. The fundamental Planck scale in the extra dimensions $\left(M_{S}\right)$, the characteristic size of the $d$ extra dimensions $(R)$ and the Planck scale on the brane are related via $M_{P l}^{2} \propto M_{S}^{d+2} R^{d}$, a purely classical relationship calculated by applying the $4+d$ dimensional Gauss's law. In this scenario, then, the weakness of gravity compared to the other SM interactions is explained by the suppression of the gravitational field flux by a factor proportional to the volume of the extra dimensions.

While direct graviton emission cross section is well defined, the cross section for virtual graviton exchange depends on a particular representation of the interaction Lagrangian and the definition of the ultraviolet cutoff on the KK modes. Three such representations have appeared nearly simultaneously [2, 3, 4]. In all of them, the effects of ED are parameterized via a single variable $\eta_{G}=\mathscr{F} / M_{S}^{4}$, where $\mathscr{F}$ is a dimensionless parameter of order one reflecting the dependence of virtual $G_{\mathrm{KK}}$ exchange on the number of extra dimensions, and $M_{S}$ is the ultraviolet cutoff. Different formalisms use different definitions of $\mathscr{F}$ [2, 3, 4], which result in different definitions of $M_{S}$.

Existing collider experimental data analysis gave no observation of LED effects, but only constraints. Indirect graviton effects at the LHC were searched for in processes of lepton and photon pair production. The corresponding constraints on $M_{S}$ (HLZ) obtained from LHC data were found to be around 5.2 TeV (ATLAS) [5] and 4.8 TeV (CMS) [6] for $d=3$.

A general feature of the different theories extending the SM of elementary particles is that new interactions involving heavy elementary objects and mass scales should exist, and manifest themselves via deviations of measured observables from the SM predictions. Here, we consider a case when the heavy intermediate states could not be produced even at the highest energy supercolliders and, correspondingly, only "virtual" effects can be expected. A description of the relevant new interaction in terms of "effective" contact-interaction (CI) is most appropriate in this case. Of course, since different interactions can give rise to similar deviations from the SM predictions, the problem is to identify, from a hypothetically measured deviation.

\section{Discovery and identification reach on $M_{S}$ in the dilepton channel}

We shall here discuss the possibility of distinguishing such effects of extra dimensions from other new physics (NP) scenarios in lepton pair production at the LHC:

$$
p+p \rightarrow l^{+} l^{-}+X
$$

where $l=e, \mu$.

At hadron colliders in the SM lepton pairs can be produced at tree-level via the following parton-level process

$$
q \bar{q} \rightarrow \gamma, Z \rightarrow l^{+} l^{-}
$$


Now, if gravity can propagate in extra dimensions, the possibility of KK graviton exchange opens up two tree-level channels in addition to the SM channels, namely

$$
q \bar{q} \rightarrow G_{n}^{*} \rightarrow l^{+} l^{-} \quad \text { and } \quad g g \rightarrow G_{n}^{*} \rightarrow l^{+} l^{-},
$$

where $G_{n}^{*}$ represents the gravitons of the KK tower.

To estimate the discovery reach of graviton towers in the ADD model one can use the invariant mass distributions of lepton pairs that have significantly different behavior in the SM and the ADD model. From a conventional $\chi^{2}$ analysis can be determined discovery reach of graviton towers in the ADD model. In particular, Fig. 1 shows discovery reach (gray band) on cutoff scale $M_{S}$ at $95 \%$ C.L. for $d=3$ and $d=6$ as a function of integrated luminosity of the LHC.

In practice the asymmetry, which is defined based on the angular distribution of the final states in scattering or decay processes, can be utilized to scrutinize underlying dynamics in NP beyond the SM. As one of the possible NP which might be discovered early at the LHC, LED are theoretical well motivated. Once LED are discovered at the LHC, it is crucial to discriminate the different NP scenarios that can lead to the same or very similar experimental signatures. In principle such task can be accomplished by measuring the angular distribution of the lepton final states which are produced via $G_{n}^{*}$-mediated processes. In the real data analysis, asymmetry is always adopted. In [7. 8, 9] center-edge asymmetry has been proposed at LHC for such kind of analysis.

The center-edge and total cross sections at the parton level can be defined as:

$$
\begin{aligned}
\hat{\sigma}_{\mathrm{CE}} & \equiv\left[\int_{-z^{*}}^{z^{*}}-\left(\int_{-1}^{-z^{*}}+\int_{z^{*}}^{1}\right)\right] \frac{d \hat{\sigma}}{d z} d z, \\
\hat{\sigma} & \equiv \int_{-1}^{1} \frac{d \hat{\sigma}}{d z} d z,
\end{aligned}
$$

where $z=\cos \hat{\theta}$, with $\hat{\theta}$ the angle, in the c.m. frame of the two leptons, between the lepton and the proton. Here, $0<z^{*}<1$ is a parameter which defines the border between the "center" and the "edge" regions.

The center-edge asymmetry at hadron level for a given dilepton invariant mass $M_{l l}$ can be defined as

$$
A_{\mathrm{CE}}\left(M_{l l}\right)=\frac{d \sigma_{\mathrm{CE}} / d M_{l l}}{d \sigma / d M_{l l}}
$$

where a convolution over parton momenta is performed, and we obtain $d \sigma_{\mathrm{CE}} / d M_{l l}$ and $d \sigma / d M_{l l}$ from the inclusive differential cross sections $d \sigma_{\mathrm{CE}} / d M_{l l} d y d z$ and $d \sigma / d M_{l l} d y d z$, respectively, by integrating over $z$ according to Eq. (2.4) and over rapidity $y$ between $-Y$ and $Y$, with $Y=$ $\log \left(\sqrt{s} / M_{l l}\right)$.

For the SM contribution to the center-edge asymmetry, the convolution integrals, depending on the parton distribution functions, cancel, and one finds

$$
A_{\mathrm{CE}}^{\mathrm{SM}}=\frac{1}{2} z^{*}\left(z^{* 2}+3\right)-1 .
$$

This result is thus independent of the dilepton mass $M_{l l}$, and identical to the result for $e^{+} e^{-}$colliders. Hence, in the case of no cuts on the angular integration, there is a unique value, $z^{*}=z_{0}^{*} \simeq 0.596$, for which $A_{\mathrm{CE}}^{\mathrm{SM}}$ vanishes, corresponding to $\hat{\theta}=53.4^{\circ}$. 
The SM center-edge asymmetry of Eq. (2.6) is equally valid for a wide variety of NP models: composite-like contact interactions, heavy $Z^{\prime}$ bosons [10], TeV-scale gauge bosons, etc. However, if graviton tower exchange is possible, the graviton tensor couplings would yield a different angular distribution, leading to a different dependence of $A_{\mathrm{CE}}$ on $z^{*}$. In this case, the center-edge asymmetry would not vanish for the above choice of $z^{*}=z_{0}^{*}$. Furthermore, it would show a non-trivial dependence on $M_{l l}$. Thus, a value for $A_{\mathrm{CE}}$ different from $A_{\mathrm{CE}}^{\mathrm{SM}}$ would indicate non-vector-exchange of NP.

Another important difference from the SM case and NP CI-like scenarios is that the graviton also couples to gluons, and therefore it has the additional $g g$ initial state of Eq. (2.3) available. In summary then, including graviton exchange and also experimental cuts relevant to the LHC detectors, the center-edge asymmetry is no longer the simple function of $z^{*}$ given by Eq. (2.6).

We assume now that a deviation from the SM is discovered in the cross section in the form of "effective" CI. We will here investigate in which regions of the ADD parameter spaces such a deviation can be identified as being caused by spin-2 exchange. More precisely, we will see how the center-edge asymmetry (2.5) can be used to exclude spin-1 exchange interactions beyond that of the SM.

From a conventional $\chi^{2}$ analysis we can find the ADD-scenario identification reach on $M_{S}$ at the LHC. The results are summarized in Fig. 1 which shows the identification reaches for different number of extra dimensions $(d=3,6)$ as a function of integrated luminosity $\mathscr{L}_{\text {int }}$.

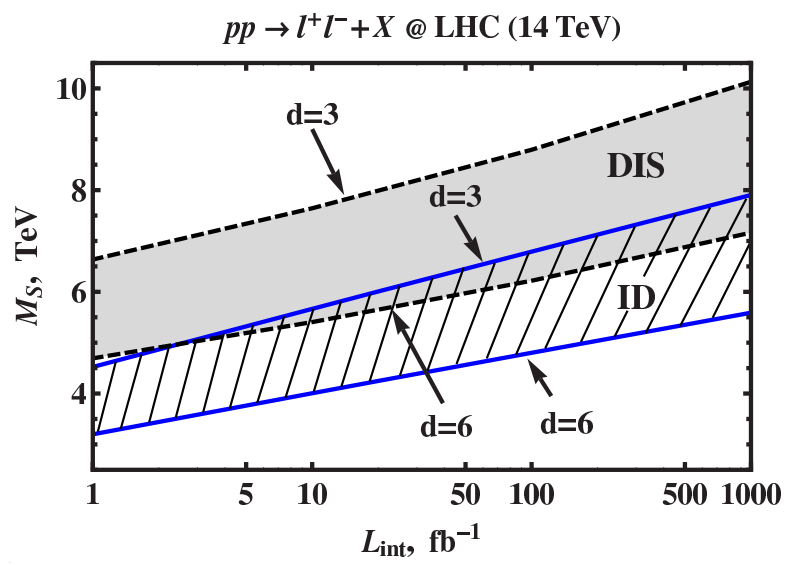

Figure 1: Discovery (gray band) and identification (hatched band) reaches on $M_{S}$ (in TeV) at 95\% CL as a function of integrated luminosity $\mathscr{L}_{\text {int }}$ for different number of extra dimensions $(d=3-6)$ at the LHC with $14 \mathrm{TeV}$.

A method proposed here and based on $A_{\mathrm{CE}}$ is suitable for actually pinning down the spin-2 nature of the KK gravitons up to very high $M_{S}$ close to discovery reach. Therefore, the analysis sketched here can potentially represent a valuable method complementary to the direct fit to the angular distribution of the lepton pairs. We find that for $\sqrt{s}=14 \mathrm{TeV}$ and $\mathscr{L}_{\text {int }}=100 \mathrm{fb}^{-1}$ the LHC detectors will be capable of discovering and identifying graviton spin-2 exchange effects in the ADD scenario with $M_{S}^{D I S}=6.2 \mathrm{TeV}\left(M_{S}^{I D}=4.8 \mathrm{TeV}\right)$ for $d=6$ and $M_{S}^{D I S}=8.8 \mathrm{TeV}\left(M_{S}^{I D}=6.8\right.$ $\mathrm{TeV}$ ) for $d=3$. 


\section{Discovery and identification reach on $M_{S}$ in the diphoton channel}

The process of photon pairs production

$$
p+p \rightarrow \gamma \gamma+X
$$

is one of the important processes at the hadron colliders and has been used to do precision of the SM. Also it provides a laboratory for probing new physics (CI, unparticles, supersymmetry, extra dimensions, etc.). A unique feature of the process of photon pairs production in the ADD model compared with Drell - Yan process is that intermediate states in this process can only be scalar and tensor particles whereas in dileptonic production does not exclude the possibility of the existence vector state. The Landau-Yang theorem [11, 12] forbids decays of vector particle into two photons. As an intermediate state we consider the scalar unparticle [13, 14]. Reducing the number of hypothetical intermediate states in the Born approximation effectively leads to "enhance" the sensitivity of the observed values for dynamic parameters of graviton tower and, thereby, expands the identification reach the effects of graviton tower exchange in the ADD model.

Discovery reach on $M_{S}$ in the diphoton channel can be determined from a conventional $\chi^{2}$ analysis. Fig. 2 shows discovery reach (gray band) on cutoff scale $M_{S}$ at $95 \%$ C.L. for $d=3$ and $d=6$ as a function of integrated luminosity of the LHC.

The purpose of this analysis is to determine the range of variation scale parameter $M_{S}$ (for a fixed $d$ ), where the ADD model (called the "correct" model) can be statistically distinguish from others competing models NP with alternative physical nature (in this case from the unparticle) hereinafter referred to the "test" models, for any value of their parameters that could mimic the effects of experimental the "correct" model. In this case, the limit on parameter $M_{S}$ is called the identification reach. To discriminate the effects of "correct" and "test" models can be define from a conventional $\chi^{2}$ analysis. Results of numerical analysis for discovery (DIS) and identification reach (ID) are shown in Fig. 2 .

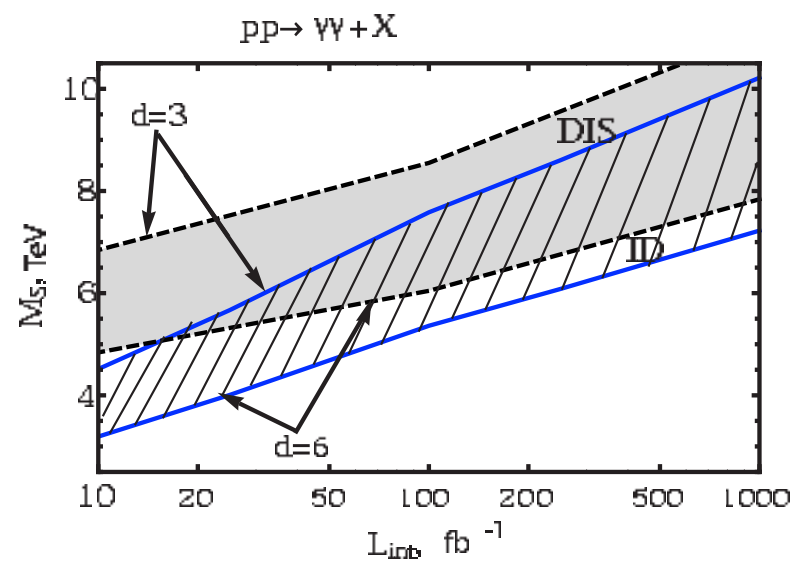

Figure 2: Discovery (gray band) and identification (hatched band) reaches on $M_{S}$ (in TeV) at 95\% CL as a function of integrated luminosity $\mathscr{L}_{\text {int }}$ for different number of extra dimensions $(d=3-6)$ at the LHC with $14 \mathrm{TeV}$. 


\section{Conclusion}

The effects of the graviton towers exchange in the ADD model as like as a CI can be found among the first effect of NP at the Large Hadron Collider. The Drell - Yan process of lepton pairs production is one of the most effective search for new channels of intermediate states due to the strong suppression of the background processes and high efficiency identification of lepton pairs. This is largely true for the process of photon pairs production. If any indirect effects of NP are found in dilepton and/or diphoton channels, such as the deviation of the distribution the invariant mass of the lepton or photon pairs from the predictions of SM, the next step of the study of the nature of this new phenomenon is to determine the spin of the intermediate state. We have investigated the prospects of experimental search for and identification of indirect effects of the exchange of graviton KK tower which existence is predicted by the ADD model in the process of lepton and photon pairs in the ATLAS experiment at the LHC. Search these new effects can by found by the specific behavior of the distribution of the lepton and photon pairs. A spin identification intermediate states can be performed by the center - edge assymetry. The results of numerical analysis (identification and discovery reach) effects of extra spatial dimensions in dilepton and diphoton channels are shown in Table. 1 .

Table 1: Discovery and identification reach on $M_{S}($ in $\mathrm{TeV}$ ) at the LHC

\begin{tabular}{|c|c|c|}
\hline $\begin{array}{c}M_{S} \\
(\mathrm{TeV})\end{array}$ & $\begin{array}{c}l^{+} l^{-} \\
\text {DIS (ID) }\end{array}$ & $\begin{array}{c}\gamma \gamma \\
\text { DIS (ID) }\end{array}$ \\
\hline$d=3$ & $8.8(6.8)$ & $8.5(7.6)$ \\
$d=6$ & $6.2(4.8)$ & $6.0(5.4)$ \\
\hline
\end{tabular}

\section{References}

[1] N. Arkani-Hamed, S. Dimopoulos, and G. Dvali, Phys. Lett. B 429, 263 (1998).

[2] J. L. Hewett, Phys. Rev. Lett. 82, 4765 (1999).

[3] G. F. Giudice, R. Rattazzi and J. D. Wells, Nucl. Phys. B 544, 3 (1999).

[4] T. Han, J. Lykken and R. Zhang, Phys. Rev. D 59, 105006 (1999).

[5] G. Aad et al. [ATLAS Collaboration], Phys. Rev. D 87, 015010 (2013).

[6] S. Chatrchyan et .al. [CMS Collaboration], CMS-PAS-EXO-12-031 (2013).

[7] P. Osland, A. A. Pankov and N. Paver, Phys. Rev. D 68, 015007 (2003).

[8] E. W. Dvergsnes, P. Osland, A. A. Pankov and N. Paver, Phys. Rev. D 69, 115001 (2004).

[9] P. Osland, A. A. Pankov, N. Paver, A.V. Tsytrinov, Phys. Rev. D 78, 035008 (2008).

[10] A. Gulov and V. Skalozub, Int. J. Mod. Phys. A 25, 5787 (2010).

[11] L. D. Landau, Dokl. Akad. Nauk. SSSR, Vol. 60, P. 207 (1948).

[12] C. N. Yang, Phys. Rev. Vol. 77, P. 242 (1950).

[13] H. Georgi, Phys. Rev. Lett. 98, 221601 (2007).

[14] H. Georgi, Phys. Lett. B 650, 275 (2007). 